\title{
ON THE OSCILLATION OF SECOND-ORDER NONLINEAR DIFFERENTIAL EQUATIONS WITH DAMPING
}

\author{
SÜLEYMAN ÖĞREKÇI, ADIL MISIR, AND AYDIN TIRYAKI
}

Received 16 December, 2014

\begin{abstract}
In this paper, we are concerned with the oscillations in forced second order nonlinear differential equations with nonlinear damping terms. By using clasical variational principle and averaging technique, new oscillation criteria are established, which revise, improve and extend some recent results. Furthermore our study answers the comment [16]. Examples are also given to illustrate the results.
\end{abstract}

2010 Mathematics Subject Classification: 34C10; 34C15; 34K11

Keywords: differential equations, oscillation, damping

\section{INTRODUCTION}

Consider the second-order nonlinear forced differential equation for $t \geq t_{0}>0$

$$
\left(r(t) k_{1}\left(x, x^{\prime}\right)\right)^{\prime}+p(t) k_{2}\left(x, x^{\prime}\right) x^{\prime}+q(t) f(x)=e(t),
$$

where $p, q \in C\left(\left[t_{0}, \infty\right), \mathbb{R}\right), r \in C^{1}\left(\left[t_{0}, \infty\right),(0, \infty)\right), f, e \in C(\mathbb{R}, \mathbb{R})$, $k_{1} \in C^{1}\left(\mathbb{R}^{2}, \mathbb{R}\right)$ and $k_{2} \in C\left(\mathbb{R}^{2}, \mathbb{R}\right)$. We restrict our attention to solutions of Eq. (1.1) which exists on $\left[t_{0}, \infty\right)$. As usual, such a solution, $x(t)$, is said to be oscillatory if it has arbitrarily zeros for all $t_{0} \geq 0$, otherwise, it is called nonoscillatory. Eq. (1.1) is called oscillatory if all solutions are oscillatory.

In the last decades, there has been an increasing interest in obtaining sufficient conditions for the oscillation and nonoscillation of solutions for different classes of second-order nonlinear forced (unforced) differential equations with damping see, for instance, [1,3, 5-8, 12-15, 17, 18, 20, 21, 23].

Many results are established for the particular cases of Eq. (1.1) ; for example, Wong [20] studied the equation for $t \geq t_{0}$

$$
x^{\prime \prime}(t)+p(t) x^{\prime}(t)+q(t) f(x(t))=0 .
$$

In [15,21], the authors obtained oscillation criteria for the equation for $t \geq t_{0}$

$$
\left(r(t) x^{\prime}(t)\right)^{\prime}+p(t) x^{\prime}(t)+q(t) f(x(t))=0 .
$$


Tiryaki and Zafer [17], Mustafa, Rogovchenko and Rogovchenko [12] obtained several oscillation results for the nonlinear equation for $t \geq t_{0}$,

$$
\left(r(t) \psi(x(t)) x^{\prime}(t)\right)^{\prime}+p(t) x^{\prime}(t)+q(t) f(x(t))=0 .
$$

The general nonlinear differential equation with damping for $t \geq t_{0}$

$$
\left(r(t) k\left(x(t), x^{\prime}(t)\right) x^{\prime}(t)\right)^{\prime}+p(t) k\left(x(t), x^{\prime}(t)\right) x^{\prime}(t)+q(t) f(x(t))=0,
$$

has been considered recently by Ayanlar and Tiryaki [1] and S. P. Rogovchenko, Yu. V. Rogovchenko [13].

The more general nonlinear differential equation with damping for $t \geq t_{0}$,

$$
\left(r(t) k_{1}\left(x(t), x^{\prime}(t)\right)\right)^{\prime}+p(t) k_{2}\left(x(t), x^{\prime}(t)\right) x^{\prime}(t)+q(t) f(x(t))=0
$$

has been first studied by Rogovchenko and Rogovchenko [14], which coincides our main equation with $e(t)=0$. Later, Rogovchenko's study [14] has been extended by Tiryaki and Zafer [19]. They obtained several oscillation criteria for solution of Eq. (1.6) under some relationships between the functions $k_{1}$ and $k_{2}$.

In 2006, Zhao and Meng [22] obtained some oscillation results for the nonlinear differential equation Eq. (1.6) They established new oscillation criteria which are extension and generalization of some known results by using the Riccati technique and the kernel functions of Philos' type under the following assumptations

(A1) $p(t) \geq 0$ for all $t \geq t_{0}, x f(x)>0$ for all $x \neq 0$;

(A2) $v k_{1}(u, v) \geq \beta_{1}\left|k_{1}(u, v)\right|^{\frac{\alpha+1}{\alpha}}$ for some $\beta_{1}>0$, and all $(u, v) \in \mathbb{R}^{2}$;

(A3) $v k_{2}(u, v) f^{\frac{1}{\alpha}}(u) \geq \beta_{2}\left|k_{1}(u, v)\right|^{\frac{\alpha+1}{\alpha}}$ for some $\beta_{2}>0$, and all $(u, v) \in \mathbb{R}^{2}$;

(A4) $f^{\prime}(x)$ exists and $\frac{f^{\prime}(x)}{|f(x)|^{\frac{\alpha-1}{\alpha}}} \geq \beta_{3}>0$ for some positive constant $\beta_{3}$ and for all $x \in \mathbb{R} \backslash\{0\}$;

or

(A5) $q(t) \geq 0$ for all $t \geq t_{0}, f$ satisfies $\frac{f(x)}{x} \geq L$ for for some positive constant $L$ and for all $x \neq 0$;

(A6) $v k_{1}(u, v) \geq \beta_{4}\left|k_{1}(u, v)\right|^{\frac{\alpha+1}{\alpha}} u^{\frac{\alpha-1}{\alpha}}$ for some positive constant $\beta_{4}$ and for all $v \in \mathbb{R} \backslash\{0\}$ and all $u \in \mathbb{R}$;

(A7) $v u^{\frac{1}{\alpha}} k_{2}(u, v) \geq \beta_{5}\left|k_{1}(u, v)\right|^{\frac{\alpha+1}{\alpha}}$ for some positive constant $\beta_{5}$ and for all $(u, v) \in \mathbb{R}^{2}$.

In 2007, Çakmak and Tiryaki [2] shoved that the proof given by Zhao and Meng [22] are inaccurate when $x(t)<0$ for $t \geq t_{0}$, because if we take $x(t)<0$ then by assumptations (A1) and (A5) $f(x)$ becomes negative, therefore (A3), (A6) and (A7) are not satisfied. Thus, although, Zhao and Meng's study [22] is very interesting and well-organized, there are some important mistakes. Therefore, Çakmak and Tiryaki [2] suggested to change the conditions (A3), (A6) and (A7) by replacing with (A3a), (A6a) and (A7a) such that

(A3a) $v k_{2}(u, v)|f(u)|^{\frac{1}{\alpha}} \geq \beta_{2}\left|k_{1}(u, v)\right|^{\frac{\alpha+1}{\alpha}}$ for some $\beta_{2}>0$, and all $(u, v) \in \mathbb{R}^{2}$; 
(A6a) $v k_{1}(u, v) \geq \beta_{4}\left|k_{1}(u, v)\right|^{\frac{\alpha+1}{\alpha}}|u|^{\frac{\alpha-1}{\alpha}}$ for some positive constant $\beta_{4}$, and for all $v \in \mathbb{R} \backslash\{0\}$ and all $u \in \mathbb{R}$;

(A7a) $v|u|^{\frac{1}{\alpha}} k_{2}(u, v) \geq \beta_{5}\left|k_{1}(u, v)\right|^{\frac{\alpha+1}{\alpha}}$ for some positive constant $\beta_{5}$ and for all $(u, v) \in \mathbb{R}^{2}$.

In 2008, Huang and Meng [9], take into considerations of Çakmak and Tiryaki's paper [2] and obtained some oscillation results for the nonlinear equation Eq. (1.6) under the same assumptations (A1)-(A7) in Zhao and Meng's paper [21] but replace the conditions (A3), (A6) and (A7) with (A3a), (A6a) and (A7a).

In 2011, Shang and Qin [16] showed that if Huang and Meng's conditions are taken into consideration there exists restriction on $f$ and $k_{1}$ due to (A4) $f(u)$ and $k_{1}$ becomes $f(u) \geq\left(\frac{\beta_{3}}{\alpha}\right)^{\alpha}|u|^{\alpha}$ and $\left|k_{1}(u, v)\right|=\beta_{1}^{-\alpha}|v|^{\alpha}$ by the assumptation (A2) and by the choice of $v k_{1}(u, v)=\beta_{1}\left|k_{1}(u, v)\right|^{\frac{\alpha+1}{\alpha}}$. Then they showed that if (A3a) holds and letting $f(u)=\left(\frac{\beta_{3}}{\alpha}\right)^{\alpha}|u|^{\alpha-1} u, k_{1}(u, v)=|v|^{\alpha-1} v$, we obtain $v k_{2}(u, v) \geq$ $\beta_{2} \beta_{3}^{-1}|v|^{\frac{\alpha+1}{\alpha}}|u|^{-1}$. On the other hand $k_{2}$ become discontinuous at $u=0$, which leads to contradiction by the assumptation of $k_{2}$. Additionally, in their Example 5.2 in [9], the outhors considered the equation

$$
\left(r(t) k_{1}\left(x, x^{\prime}\right)\right)^{\prime}+p(t) k_{2}\left(x, x^{\prime}\right) x^{\prime}+q(t) f(x)=0,
$$

with

$$
k_{1}(u, v)=|v|^{p-1} v \quad \text { and } \quad k_{2}(u, v)=\frac{|v|^{p-1} v}{u\left(1+u^{2}\right)^{\frac{1}{p}}} .
$$

But Shang and Qin [16] also showed that

$$
v k_{2}(u, v)=\frac{|v|^{p-1} v^{2}}{u\left(1+u^{2}\right)^{\frac{1}{p}}}
$$

is discontinuous at $u=0$ and

$$
k_{1}(u, v)=|v|^{p-1} v
$$

is not continuously differentiable at $v=0$. Therefore, oscillatory solutions of the given equation in Example 5.2 in [9] does not exist. Thus it seems that the conditions (A3a), (A6a) and (A7a) still need reconsideration.

Since the conditions of [9] used in the proofs, the recent papers [11] and [10] also need revisement. Motivated by this fact, in this paper, first we will investigate the oscillatory behavior of second-order nonlinear forced differential equation Eq. (1.1) by revising the conditions (A3a), (A6a) and (A7a) to overcome the difficulties that we mentioned above. Secondly, we define a new form of the functional $A_{s_{i}}^{t_{i}}(h ; t)$ as $A_{s_{i}}^{t_{i}}(h ; \alpha+1)$. By this way we overcome the problems of the singularity of the functional $A_{s_{i}}^{t_{i}}(h ; t)$ at the points $s_{i}, t_{i}(i=1,2)$ and the problems of the inapplicability of 
the functional $A_{s_{i}}^{t_{i}}(h ; t)$ for some $\alpha$ as mentioned in Theorem 2.1 in [20], again Theorem 2.1 in [2] and theorem 1.2 in [15] and so forth. Finally we give some illustrated examples.

\section{MAIN RESUltS}

Firstly we introduce the general mean and some well known properties that will be used in the proofs of our results.

Let

$$
D\left(s_{i}, t_{i}\right)=\left\{u \in C^{1}\left[s_{i}, t_{i}\right]: u(t) \neq 0 \text { for } t \in\left(s_{i}, t_{i}\right), u\left(s_{i}\right)=u\left(t_{i}\right)=0\right\},
$$

for $i=1,2$. We define the functional $A_{s_{i}}^{t_{i}}(\cdot ; n)$ for $H \in D\left(s_{i}, t_{i}\right)$ and $n \geq 0$ such as;

$$
A_{s_{i}}^{t_{i}}(h ; n)=\int_{s_{i}}^{t_{i}}|H(t)|^{n} h(t) d t, \quad s_{i} \leq t \leq t_{i}, i=1,2,
$$

where $h \in C\left(\left[t_{0}, \infty\right),[0, \infty)\right)$. It is easily seen that the linear functional $A_{s_{i}}^{t_{i}}(\cdot ; n)$ satisfies the conditions

(1) $A_{s_{i}}^{t_{i}}(h ; n)=A_{s_{i}}^{t_{i}}\left(|H|^{k} h ; n-k\right)$,for $i=1,2$ and $k \in \mathbb{R}$;

(2) $A_{s_{i}}^{t_{i}}\left(h^{\prime} ; n\right) \geq-A_{s_{i}}^{t_{i}}\left(n\left|H^{\prime} h\right| ; n-1\right)$, for $i=1,2$.

In this section, we shall make use of the following conditions:

$\left(B_{1}\right) f(x)$ is differentiable and $x f(x)>0$ for all $x \neq 0$,

$\left(B_{2}\right) v k_{1}(u, v) \geq \beta_{1}\left|k_{1}(u, v)\right|^{(\alpha+1) / \alpha}$, for some $\alpha>0, \beta_{1}>0$ and for all $(u, v) \in$ $\mathbb{R}^{2}$.

$\left(B_{3}\right) u v k_{2}(u, v) \geq 0$ for all $(u, v) \in \mathbb{R}^{2}$,

$\left(B_{4}\right) f^{\prime}(x)$ exists and $\frac{f^{\prime}(x)}{|f(x)|^{\frac{\alpha-1}{\alpha}}} \geq \beta_{2}>0$ for some positive constant $\beta_{2}$ and for all $x \in \mathbb{R} \backslash\{0\}$,

(B) $\frac{f(x)}{x} \geq K|x|^{\gamma-1}$ for some $K>0, \gamma \geq 1$ and for all $x \neq 0$,

(B) $v k_{1}(u, v) \geq \beta_{3}\left|k_{1}(u, v)\right|^{(\alpha+1) / \alpha}|u|^{(\alpha-1) / \alpha}$, for some $\alpha>0, \beta_{3}>0$ and for all $u \in \mathbb{R}, v \neq 0$.

Theorem 1. Suppose the conditions $\left(B_{1}\right)-\left(B_{4}\right)$ holds and for any $T \geq t_{0}$, there exists $T \leq s_{1}<t_{1} \leq s_{2}<t_{2}$ such that

$$
e(t) \leq 0 \text { for } t \in\left[s_{1}, t_{1}\right], e(t) \geq 0 \text { for } t \in\left[s_{2}, t_{2}\right] \text { and } p(t)>0 \text { on }\left[s_{1}, t_{1}\right] \cup\left[s_{2}, t_{2}\right] \text {. }
$$

Let $D\left(s_{i}, t_{i}\right)$ and $A_{s_{i}}^{t_{i}}(\cdot ; n)$ are defined by (2.1) and (2.2) respectively. If there exists $H \in D\left(s_{i}, t_{i}\right)$ and a nonnegative constant $n$ such that

$$
A_{s_{i}}^{t_{i}}(q ; n+\alpha+1)>A_{s_{i}}^{t_{i}}\left(\delta r\left|H^{\prime}\right|^{\alpha+1} ; n\right),
$$


for $i=1,2$, then Eq. (1.1) is oscillatory. Where $\delta=\left(\frac{\alpha}{\beta_{1} \beta_{2}}\right)^{\alpha}\left(\frac{n+\alpha+1}{\alpha+1}\right)^{\alpha+1}$.

Proof. On the contrary, suppose that Eq. (1.1) has a nonoscillatory solution $x(t)$. Then $x(t)$ eventually must have one sign, i.e. $x(t) \neq 0$ on $\left[T_{0}, \infty\right)$ for some large $T_{0} \geq t_{0}$. By the assumptions, there exists $s_{1}, t_{1}, s_{2}$ and $t_{2}$ such that $T_{0} \leq s_{1}<t_{1} \leq$ $s_{2}<t_{2}$ and (2.3) holds. Define

$$
w(t)=\frac{r(t) k_{1}\left(x(t), x^{\prime}(t)\right)}{f(x(t))}, t \in\left[s_{1}, t_{1}\right] \cup\left[s_{2}, t_{2}\right] .
$$

Then differentiating (2.5) and using Eq. (1.1) we obtain

$$
\begin{aligned}
w^{\prime}(t) & =-q(t)-\frac{p(t) k_{2}\left(x(t), x^{\prime}(t)\right) f(x(t)) x^{\prime}(t)}{f^{2}(x(t))} \\
& -\frac{r(t) k_{1}\left(x(t), x^{\prime}(t)\right) x^{\prime}(t) f^{\prime}(x(t))}{f^{2}(x(t))}+\frac{e(t)}{f(x(t))} .
\end{aligned}
$$

By using assumptions $\left(B_{1}\right)-\left(B_{4}\right)$ we obtain for $t \in\left[s_{1}, t_{1}\right] \cup\left[s_{2}, t_{2}\right]$

$$
w^{\prime}(t) \leq-q(t)-\frac{\beta_{1} \beta_{2}}{r^{1 / \alpha}(t)}|w(t)|^{(\alpha+1) / \alpha}+\frac{e(t)}{f(x(t))} .
$$

On the intervals $\left[s_{1}, t_{1}\right]$ (if $x(t)>0$ ) or $\left[s_{2}, t_{2}\right]$ (if $\left.x(t)<0\right), w(t)$ satisfies

$$
w^{\prime}(t) \leq-q(t)-a(t)|w(t)|^{(\alpha+1) / \alpha},
$$

where $a(t)=\beta_{1} \beta_{2} r^{-1 / \alpha}(t)$.

Now first we assume that $x(t)>0$ on $\left[T_{0}, \infty\right)$ for some large $T_{0} \geq t_{0}$. Multiplying $|H(t)|^{n+\alpha+1}$ throughout Eq.(2.7) and integrating from $s_{i}$ to $t_{i}$ for $i=1$, we obtain

$$
A_{s_{1}}^{t_{1}}(q ; n+\alpha+1) \leq A_{s_{1}}^{t_{1}}\left((n+\alpha+1)|H|^{\alpha}\left|H^{\prime}\right||w|-a|H|^{\alpha+1}|w|^{(\alpha+1) / \alpha} ; n\right),
$$

where $D\left(s_{i}, t_{i}\right)$ is given by hypotheses. Setting

$$
F(v):=(n+\alpha+1)|H|^{\alpha}\left|H^{\prime}\right| v-a|H|^{\alpha+1} v^{(\alpha+1) / \alpha}, \quad v>0,
$$

we have $F^{\prime}\left(v^{*}\right)=0$ and $F^{\prime \prime}\left(v^{*}\right)<0$, where $v^{*}=\left(\frac{\alpha(n+\alpha+1)}{\alpha+1} \frac{1}{a}\left|\frac{H^{\prime}}{H}\right|\right)^{\alpha}$, which implies that $F(v)$ obtains its maximum at $v^{*}$. So we have

$$
F(v) \leq F\left(v^{*}\right)=\left(\frac{\alpha}{a}\right)^{\alpha}\left(\frac{n+\alpha+1}{\alpha+1}\right)^{\alpha+1}\left|H^{\prime}\right|^{\alpha+1} .
$$

Then we get, by using (2.9) in (2.8)

$$
A_{s_{i}}^{t_{i}}(q ; n+\alpha+1) \leq A_{s_{i}}^{t_{i}}\left(\delta r\left|H^{\prime}\right|^{\alpha+1} ; n\right),
$$

which contradicts to (2.4) for $i=1$.

If $x(t)<0$ on $\left[T_{0}, \infty\right)$ for some large $T_{0} \geq t_{0}$, we get the inequality (2.6) again, which implies that (2.7) holds on the interval $\left[s_{2}, t_{2}\right]$. Applying operator $A_{s_{i}}^{t_{i}}(\cdot ; n)$ for 
$i=2$ as mentioned above to (2.7) this time, we get the same contradiction to (2.4) for $i=2$. Thus the proof is complete.

Lemma 1. [4]If $A$ and $B$ are non-negative constants and $m, n \in \mathbb{R}$ such that $\frac{1}{m}+\frac{1}{n}=1$, then

$$
\frac{1}{m} A+\frac{1}{n} B \geq A^{1 / m} B^{1 / n}
$$

Theorem 2. Suppose the conditions $\left(B_{1}\right),\left(B_{3}\right),\left(B_{5}\right),\left(B_{6}\right)$ holds and for any $T \geq t_{0}$, there exists $T \leq s_{1}<t_{1} \leq s_{2}<t_{2}$ such that condition (2.3) holds and $q(t) \geq 0$ on $\left[s_{1}, t_{1}\right] \cup\left[s_{2}, t_{2}\right]$. If there exists a $H \in D\left(s_{i}, t_{i}\right)$ and a nonnegative constant $n$ such that

$$
A_{s_{i}}^{t_{i}}(Q ; n+\alpha+1)>A_{s_{i}}^{t_{i}}\left(\delta_{1} r\left|H^{\prime}\right|^{\alpha+1} ; n\right),
$$

for $i=1,2$, where

$$
Q(t)=\gamma(\gamma-1)^{(1-\gamma) / \gamma}[K q(t)]^{1 / \gamma}|e(t)|^{(\gamma-1) \gamma}, \delta_{1}=\left(\frac{\alpha}{\beta_{3}}\right)^{\alpha}\left(\frac{n+\alpha+1}{\alpha+1}\right)^{\alpha+1},
$$

with the convention $0^{0}=1$. Then Eq. (1.1) is oscillatory.

Proof. On the contrary, suppose that Eq. (1.1) has a nonoscillatory solution $x(t)$. Then $x(t)$ eventually must have one sign. First we assume that $x(t)>0$ on $\left[T_{0}, \infty\right)$ for some large $T_{0} \geq t_{0}$. Define

$$
\theta(t)=\frac{r(t) k_{1}\left(x(t), x^{\prime}(t)\right)}{x(t)}, t \in\left[s_{1}, t_{1}\right] \cup\left[s_{2}, t_{2}\right] .
$$

Then differentiating (2.12) and using Eq. (1.1) we obtain

$$
\begin{aligned}
\theta^{\prime}(t) & =-\frac{q(t) f(x(t))}{x(t)}-\frac{p(t) k_{2}\left(x(t), x^{\prime}(t)\right) x(t)}{x^{2}(t)} \\
& -\frac{r(t) k_{1}\left(x(t), x^{\prime}(t)\right) x^{\prime}(t)}{x^{2}(t)}+\frac{e(t)}{x(t)} .
\end{aligned}
$$

By using $\left(B_{1}\right),\left(B_{2}\right),\left(B_{3}\right),\left(B_{6}\right)$ and Eq. (2.3) we obtain for $t \in\left[s_{1}, t_{1}\right] \cup\left[s_{2}, t_{2}\right]$,

$$
\theta^{\prime}(t) \leq-q(t) K|x(t)|^{\gamma-1}-\frac{\beta_{3}}{r^{1 / a}(t)}|\theta(t)|^{(\alpha+1) / \alpha}+\frac{e(t)}{x(t)} .
$$

On the interval $\left[s_{1}, t_{1}\right]$, inequality (2.13) implies that $\theta(t)$ satisfies

$$
q(t) K|x(t)|^{\gamma-1}+\left|\frac{e(t)}{x(t)}\right| \leq-\theta^{\prime}(t)-\frac{\beta_{3}}{r^{1 / a}(t)}|\theta(t)|^{(\alpha+1) / \alpha} .
$$


For $\gamma>1$, by setting $m=\gamma, n=\gamma /(\gamma-1), A=\gamma K q(t)|x(t)|^{\gamma-1}$, $B=\left(\frac{\gamma}{\gamma-1}\right)\left|\frac{e(t)}{x(t)}\right|$ and using Lemma 1, we obtain

$$
q(t) K|x(t)|^{\gamma-1}+\left|\frac{e(t)}{x(t)}\right| \geq Q(t) .
$$

Hence, on the interval $\left[s_{1}, t_{1}\right], \theta(t)$ satisfies

$$
\theta^{\prime}(t) \leq-Q(t)-\frac{\beta_{3}}{r^{1 / a}(t)}|\theta(t)|^{(\alpha+1) / \alpha} .
$$

Note that the inequality holds for $\gamma=1$ also with the convention $0^{0}=1$.

Applying operator $A_{s_{i}}^{t_{i}}(\cdot ; n)$ for $i=1$, to (2.15) we obtain a contradiction to (2.11), this part of the proof is similar to Theorem 1 and hence omitted.

If $x(t)<0$ on $\left[T_{0}, \infty\right)$ for some large $T_{0} \geq t_{0}$, it is easy to see that (2.16) holds for $t \in\left[s_{2}, t_{2}\right]$. Then applying operator $A_{s_{i}}^{t_{i}}(\cdot ; n)$ for $i=2$, we still obtain contradiction. Thus the proof is complete.

Now we will give some other oscillation criteria for (1.1) through some new averaging functions $G(t, s) \in C\left(D_{1}, \mathbb{R}\right)$, which satisfy:

(i) $G(t, t)=0, G(t, s)>0$ for $t>s$,

(ii) $G$ has partial derivatives $\partial G / \partial t$ and $\partial G / \partial s$ on $D_{1}$ such that

$$
\frac{\partial G}{\partial t}=g_{1}(t, s) \sqrt{G(t, s)}, \quad \frac{\partial G}{\partial s}=-g_{2}(t, s) \sqrt{G(t, s)}
$$

where $D_{1}=\left\{(t, s): t_{0} \leq s \leq t<\infty\right\}$ and $g_{1}, g_{2} \in L_{l o c}\left(D_{1}, \mathbb{R}^{+}\right)$.

Theorem 3. Suppose the conditions $\left(B_{1}\right)-\left(B_{4}\right)$ holds and for any $T \geq t_{0}$, there exists $T \leq s_{1}<t_{1} \leq s_{2}<t_{2}$ such that condition (2.3) holds. If there exist some $\varepsilon_{i} \in$ $\left(s_{i}, t_{i}\right), i=1,2, G(t, s)$ satisfying $(i)$-(ii) and a positive function $\rho \in C^{1}\left(\left[t_{0}, \infty\right), \mathbb{R}^{+}\right)$ such that

$$
\begin{aligned}
& \frac{1}{G^{\alpha+1}\left(\varepsilon_{i}, s_{i}\right)} \int_{s_{i}}^{\varepsilon_{i}}\left[G^{\alpha+1}\left(\tau, s_{i}\right) q(\tau) \rho(\tau)-\delta_{2} G_{1}^{\alpha+1}\left(\tau, s_{i}\right) r(\tau) \rho(\tau)\right] d \tau \\
& +\frac{1}{G^{\alpha+1}\left(t_{i}, \varepsilon_{i}\right)} \int_{\varepsilon_{i}}^{t_{i}}\left[G^{\alpha+1}\left(t_{i}, \tau\right) q(\tau) \rho(\tau)-\delta_{2} G_{2}^{\alpha+1}\left(t_{i}, \tau\right) r(\tau) \rho(\tau)\right] d \tau \\
& >0
\end{aligned}
$$

for $i=1,2$ where

$$
\delta_{2}=\frac{\alpha^{\alpha}}{\beta_{1}^{\alpha} \beta_{2}^{\alpha}(\alpha+1)^{\alpha+1}},
$$




$$
\begin{aligned}
& G_{1}(t, s)=\left|(\alpha+1) g_{1}(t, s) \sqrt{G(t, s)}+G(t, s) \frac{\rho^{\prime}(s)}{\rho(s)}\right|, \\
& G_{2}(t, s)=\left|(\alpha+1) g_{2}(t, s) \sqrt{G(t, s)}-G(t, s) \frac{\rho^{\prime}(s)}{\rho(s)}\right| .
\end{aligned}
$$

Then Eq. (1.1) is oscillatory.

Proof. On the contrary, suppose that Eq. (1.1) has a nonoscillatory solution $x(t)$. Then $x(t) \neq 0$ on $[T, \infty)$ for some sufficiently large $T \geq t_{0}$. Define

$$
w_{1}(t)=\rho(t) \frac{r(t) k_{1}\left(x(t), x^{\prime}(t)\right)}{f(x(t))}, t \in\left[s_{1}, t_{1}\right] \cup\left[s_{2}, t_{2}\right] .
$$

Differentiating (2.18), using conditions $\left(B_{1}\right)-\left(B_{4}\right)$ and Eq. (1.1) we obtain for $t \in\left[s_{1}, t_{1}\right] \cup\left[s_{2}, t_{2}\right]$

$$
\begin{gathered}
w_{1}^{\prime}(t) \leq \\
-q(t) \rho(t)+\frac{\rho^{\prime}(t)}{\rho(t)} w_{1}(t)-\beta_{1} \beta_{2} r^{-1 / \alpha}(t) \rho^{-1 / \alpha}(t)\left|w_{1}(t)\right|^{(\alpha+1) / \alpha}+\frac{e(t)}{f(x(t))} \rho(t) .
\end{gathered}
$$

This implies that on the intervals $\left[s_{1}, t_{1}\right]$ (if $x(t)>0$ ) or $\left[s_{2}, t_{2}\right]$ (if $\left.x(t)<0\right), w_{1}(t)$ satisfies the inequality

$$
w_{1}^{\prime}(t) \leq-q(t) \rho(t)+\frac{\rho^{\prime}(t)}{\rho(t)} w_{1}(t)-\beta_{1} \beta_{2} r^{-1 / \alpha}(t) \rho^{-1 / \alpha}(t)\left|w_{1}(t)\right|^{(\alpha+1) / \alpha} .
$$

On the one hand, multiplying (2.19) with $G^{\alpha+1}(t, s)$ and integrating (with $t$ replaced by $s$ ) over $\left[\varepsilon_{i}, t\right)$ for $t \in\left[\varepsilon_{i}, t_{i}\right)$ and $i=1,2$ we have

$$
\begin{gathered}
\int_{\varepsilon_{i}}^{t} G^{\alpha+1}(t, s) q(s) \rho(s) d s \leq G^{\alpha+1}\left(t, \varepsilon_{i}\right) w_{1}\left(\varepsilon_{i}\right) \\
+\int_{\varepsilon_{i}}^{t}\left[G^{\alpha}(t, s) G_{2}(t, s)\left|w_{1}(s)\right|\right] d s \\
-\int_{\varepsilon_{i}}^{t}\left[\beta_{1} \beta_{2} r^{-1 / \alpha}(t) \rho^{-1 / \alpha}(t) G^{\alpha+1}(t, s)\left|w_{1}(t)\right|^{(\alpha+1) / \alpha}\right] d s .
\end{gathered}
$$

For a given $t$ and $s$, set

$$
F(v)=G^{\alpha} G_{2} v-\beta_{1} \beta_{1} r^{-1 / \alpha} \rho^{-1 / \alpha} G^{\alpha+1} v^{(\alpha+1) / \alpha}, \quad v>0 .
$$

$F$ yields its maximum at the point $v^{*}=\left(\frac{\alpha}{\alpha+1} \frac{G_{2}}{\beta_{1} \beta_{1} G r^{-1 / \alpha} \rho^{-1 / \alpha}}\right)^{\alpha}$ and

$$
F(v) \leq F_{\max }=F\left(v^{*}\right)=\delta_{2} G_{2} r \rho .
$$


Then, by using (2.21) and letting $t \rightarrow t_{i}^{-}$in (2.20), we get

$$
\begin{gathered}
\int_{\varepsilon_{i}}^{t_{i}} G^{\alpha+1}\left(t_{i}, s\right) q(s) \rho(s) d s \\
\leq G^{\alpha+1}\left(t_{i}, \varepsilon_{i}\right) w_{1}\left(\varepsilon_{i}\right)+\delta_{2} \int_{\varepsilon_{i}}^{t_{i}} G_{2}^{\alpha+1}\left(t_{i}, s\right) r(s) \rho(s) d s .
\end{gathered}
$$

On the other hand, multiplying (2.19) with $G^{\alpha+1}(s, t)$, then integrating (with $t$ replaced by $s$ ) over $\left[t, \varepsilon_{i}\right)$ for $t \in\left[t_{i}, \varepsilon_{i}\right), i=1,2$ and using similar calculations with the proof of (2.21) we get

$$
\begin{gathered}
\int_{t}^{\varepsilon_{i}} G^{\alpha+1}\left(s, s_{i}\right) q(s) \rho(s) d s \leq \\
-G^{\alpha+1}\left(\varepsilon_{i}, s_{i}\right) w_{1}\left(\varepsilon_{i}\right)+\delta_{2} \int_{t}^{\varepsilon_{i}} G_{1}^{\alpha+1}\left(s, s_{i}\right) r(s) \rho(s) d s .
\end{gathered}
$$

Letting $t \rightarrow s_{i}^{+}$in (2.23), it follows that

$$
\begin{gathered}
\int_{s_{i}}^{\varepsilon_{i}} G^{\alpha+1}\left(s, s_{i}\right) q(s) \rho(s) d s \\
\leq-G^{\alpha+1}\left(\varepsilon_{i}, s_{i}\right) w_{1}\left(\varepsilon_{i}\right)+\delta_{2} \int_{s_{i}}^{\varepsilon_{i}} G_{1}^{\alpha+1}\left(s, s_{i}\right) r(s) \rho(s) d s .
\end{gathered}
$$

Finally, dividing (2.22) and (2.24) by $G^{\alpha+1}\left(t_{i}, \varepsilon_{i}\right)$ and $G^{\alpha+1}\left(\varepsilon_{i}, s_{i}\right)$ respectively, and then adding them, we have the desired contradiction with (2.17). Thus the proof is complete.

Corollary 1. Suppose the conditions $\left(B_{1}\right)-\left(B_{4}\right)$ holds and for any $T \geq t_{0}$, there exists $T \leq s_{1}<t_{1} \leq s_{2}<t_{2}$ such that condition (2.3) holds. If there exist some $\varepsilon_{i} \in$ $\left(s_{i}, t_{i}\right), i=1,2, G(t, s)$ satisfying $(i)$-(ii) and a positive function $\rho \in C^{1}\left(\left[t_{0}, \infty\right), \mathbb{R}^{+}\right)$ such that

$$
\begin{gathered}
\int_{s_{i}}^{\varepsilon_{i}}\left[G^{\alpha+1}\left(\tau, s_{i}\right) q(\tau) \rho(\tau)-\delta_{2} G_{1}^{\alpha+1}\left(\tau, s_{i}\right) r(\tau) \rho(\tau)\right] d \tau>0, \\
\int_{\varepsilon_{i}}^{t_{i}}\left[G^{\alpha+1}\left(t_{i}, \tau\right) q(\tau) \rho(\tau)-\delta_{2} G_{2}^{\alpha+1}\left(t_{i}, \tau\right) r(\tau) \rho(\tau)\right] d \tau>0,
\end{gathered}
$$

for $i=1,2$. Then Eq. (1.1) is oscillatory.

Theorem 4. Suppose the conditions $\left(B_{1}\right),\left(B_{3}\right),\left(B_{5}\right),\left(B_{6}\right)$ holds and for any $T \geq t_{0}$, there exists $T \leq s_{1}<t_{1} \leq s_{2}<t_{2}$ such that condition (2.3) holds and $q(t) \geq 0$ 
on $\left[s_{1}, t_{1}\right] \cup\left[s_{2}, t_{2}\right]$. If there exist some $\varepsilon_{i} \in\left(s_{i}, t_{i}\right), i=1,2, G(t, s)$ satisfying (i)-(ii) and a positive function $\rho \in C^{1}\left(\left[t_{0}, \infty\right), \mathbb{R}^{+}\right)$such that

$$
\begin{aligned}
& \frac{1}{G^{\alpha+1}\left(\varepsilon_{i}, s_{i}\right)} \int_{s_{i}}^{\varepsilon_{i}}\left[G^{\alpha+1}\left(\tau, s_{i}\right) Q(\tau) \rho(\tau)-\delta_{3} G_{1}^{\alpha+1}\left(\tau, s_{i}\right) r(\tau) \rho(\tau)\right] d \tau \\
& +\frac{1}{G^{\alpha+1}\left(t_{i}, \varepsilon_{i}\right)} \int_{\varepsilon_{i}}^{t_{i}}\left[G^{\alpha+1}\left(t_{i}, \tau\right) Q(\tau) \rho(\tau)-\delta_{3} G_{2}^{\alpha+1}\left(t_{i}, \tau\right) r(\tau) \rho(\tau)\right] d \tau \\
& >0
\end{aligned}
$$

for $i=1,2$ where

$$
\delta_{3}=\frac{\alpha^{\alpha}}{\beta_{3}^{\alpha}(\alpha+1)^{\alpha+1}},
$$

and $H_{1}, H_{2}, Q$ are defined in previous theorems. Then Eq. (1.1) is oscillatory.

Proof. On the contrary, suppose that Eq. (1.1) has a nonoscillatory solution $x(t)$. Then $x(t) \neq 0$ on $[T, \infty)$ for some sufficiently large $T \geq t_{0}$. Define

$$
\theta_{1}(t)=\rho(t) \frac{r(t) k_{1}\left(x(t), x^{\prime}(t)\right)}{x(t)}, t \in\left[s_{1}, t_{1}\right] \cup\left[s_{2}, t_{2}\right] .
$$

Differentiating (2.28), using conditions $\left(B_{1}\right),\left(B_{3}\right),\left(B_{5}\right),\left(B_{6}\right)$ and Eq. (1.1) we obtain

$$
\begin{gathered}
\rho(t)\left(K q(t)|x(t)|^{\gamma-1}+\left|\frac{e(t)}{x(t)}\right|\right) \leq \\
-\theta_{1}^{\prime}(t)+\frac{\rho^{\prime}(t)}{\rho(t)} \theta_{1}(t)-\beta_{3} r^{-1 / \alpha}(t) \rho^{-1 / \alpha}(t)\left|\theta_{1}(t)\right|^{(\alpha+1) / \alpha}
\end{gathered}
$$

for $t \in\left[s_{1}, t_{1}\right]$ or $t \in\left[s_{2}, t_{2}\right]$. By choosing $m=\gamma, n=\gamma /(\gamma-1)$,

$A=\gamma K q(t)|x(t)|^{\gamma-1}$ and $B=\gamma /(\gamma-1)|e(t) / x(t)|$ in the Lemma 1 we get

$$
\rho(t) Q(t) \leq-\theta_{1}^{\prime}(t)+\frac{\rho^{\prime}(t)}{\rho(t)} \theta_{1}(t)-\beta_{3} r^{-1 / \alpha}(t) \rho^{-1 / \alpha}(t)\left|\theta_{1}(t)\right|^{(\alpha+1) / \alpha}
$$

for $t \in\left[s_{1}, t_{1}\right]$ or $t \in\left[s_{2}, t_{2}\right]$ and for $\gamma>1$. Note that the inequality (2.29) holds trivially for $\gamma=1$.

The rest of the proof is similar with the previous theorem, hence omitted.

Corollary 2. Suppose the conditions $\left(B_{1}\right),\left(B_{3}\right),\left(B_{5}\right),\left(B_{6}\right)$ holds and for any $T \geq t_{0}$, there exists $T \leq s_{1}<t_{1} \leq s_{2}<t_{2}$ such that condition (2.3) holds and $q(t) \geq 0$ on $\left[s_{1}, t_{1}\right] \cup\left[s_{2}, t_{2}\right]$. If there exist some $\varepsilon_{i} \in\left(s_{i}, t_{i}\right), i=1,2, G(t, s)$ satisfying (i)-(ii) 
and a positive function $\rho \in C^{1}\left(\left[t_{0}, \infty\right), \mathbb{R}^{+}\right)$such that

$$
\begin{gathered}
\int_{s_{i}}^{\varepsilon_{i}}\left[G^{\alpha+1}\left(\tau, s_{i}\right) Q(\tau) \rho(\tau)-\delta_{3} G_{1}^{\alpha+1}\left(\tau, s_{i}\right) r(\tau) \rho(\tau)\right] d \tau>0, \\
\int_{\varepsilon_{i}}^{t_{i}}\left[G^{\alpha+1}\left(t_{i}, \tau\right) Q(\tau) \rho(\tau)-\delta_{3} G_{2}^{\alpha+1}\left(t_{i}, \tau\right) r(\tau) \rho(\tau)\right] d \tau>0,
\end{gathered}
$$

for $i=1,2$. Then Eq. (1.1) is oscillatory.

Now we consider the more general equation

$$
\left(r(t) k_{1}\left(x, x^{\prime}\right)\right)^{\prime}+p(t)\left[A k_{2}\left(x, x^{\prime}\right) x^{\prime}+B k_{3}\left(x, x^{\prime}\right)\right]+q(t) f(x)=e(t)
$$

for $t \geq t_{0}>0$, where $A, B$ are nonnegative constants and, $k_{3} \in C\left(\mathbb{R}^{2}, \mathbb{R}\right)$ with $u k_{3}(u, v) \geq 0$ for all $(u, v) \in \mathbb{R}^{2}$. By using same substitutions with the proofs above, it is easy to proof following results. Since all the possible proofs differ from the previous proofs with only the term $u k_{3}(u, v)$, we rely on nonnegatitivity of this term.

Theorem 5. Suppose the conditions $\left(B_{1}\right)-\left(B_{4}\right)$ holds and for any $T \geq t_{0}$, there exists $T \leq s_{1}<t_{1} \leq s_{2}<t_{2}$ such that $(2.3)$ holds. Let $D\left(s_{i}, t_{i}\right)$ and $A_{s_{i}}^{\overline{t_{i}}}(\cdot ; n)$ are defined by (2.1) and (2.2) respectively. If there exists $H \in D\left(s_{i}, t_{i}\right)$ and a nonnegative constant $n$ such that the inequality (2.4) holds for $i=1,2$, then Eq. (2.32) is oscillatory.

Theorem 6. Suppose the conditions $\left(B_{1}\right),\left(B_{3}\right),\left(B_{5}\right),\left(B_{6}\right)$ holds and for any $T \geq$ $t_{0}$, there exists $T \leq s_{1}<t_{1} \leq s_{2}<t_{2}$ such that condition (2.3) holds and $q(t) \geq 0$ on $\left[s_{1}, t_{1}\right] \cup\left[s_{2}, t_{2}\right]$. Let $D\left(s_{i}, t_{i}\right)$ and $A_{s_{i}}^{t_{i}}(\cdot ; n)$ are defined by (2.1) and (2.2) respectively. If there exists $H \in D\left(s_{i}, t_{i}\right)$ and a nonnegative constant $n$ such that the inequality (2.11) holds for $i=1,2$ with the convention $0^{0}=1$. Then Eq. (2.32) is oscillatory.

Theorem 7. Suppose the conditions $\left(B_{1}\right)-\left(B_{4}\right)$ holds and for any $T \geq t_{0}$, there exists $T \leq s_{1}<t_{1} \leq s_{2}<t_{2}$ such that condition (2.3) holds. If there exist some $\varepsilon_{i} \in$ $\left(s_{i}, t_{i}\right), i=1,2, G(t, s)$ satisfying $(i)$-(ii) and a positive function $\rho \in C^{1}\left(\left[t_{0}, \infty\right), \mathbb{R}^{+}\right)$ such that the inequality (2.17) holds for $i=1,2$. Then Eq. (2.32) is oscillatory.

Theorem 8. Suppose the conditions $\left(B_{1}\right),\left(B_{3}\right),\left(B_{5}\right),\left(B_{6}\right)$ holds and for any $T \geq t_{0}$, there exists $T \leq s_{1}<t_{1} \leq s_{2}<t_{2}$ such that condition (2.3) holds and $q(t) \geq 0$ on $\left[s_{1}, t_{1}\right] \cup\left[s_{2}, t_{2}\right]$. If there exist some $\varepsilon_{i} \in\left(s_{i}, t_{i}\right), i=1,2, G(t, s)$ satisfying (i)-(ii) and a positive function $\rho \in C^{1}\left(\left[t_{0}, \infty\right), \mathbb{R}^{+}\right)$such that the inequality (2.27) holds for $i=1,2$. Then Eq. (2.32) is oscillatory.

Corollary 3. Suppose the conditions $\left(B_{1}\right)-\left(B_{4}\right)$ holds and for any $T \geq t_{0}$, there exists $T \leq s_{1}<t_{1} \leq s_{2}<t_{2}$ such that condition (2.3) holds. If there exist some $\varepsilon_{i} \in$ $\left(s_{i}, t_{i}\right), i=1,2, G(t, s)$ satisfying $(i)$-(ii) and a positive function $\rho \in C^{1}\left(\left[t_{0}, \infty\right), \mathbb{R}^{+}\right)$ 
such that the inequalities (2.25) and (2.26) holds for $i=1,2$. Then Eq. (2.32) is oscillatory.

Corollary 4. Suppose the conditions $\left(B_{1}\right),\left(B_{3}\right),\left(B_{5}\right),\left(B_{6}\right)$ holds and for any $T \geq t_{0}$, there exists $T \leq s_{1}<t_{1} \leq s_{2}<t_{2}$ such that condition (2.3) holds and $q(t) \geq 0$ on $\left[s_{1}, t_{1}\right] \cup\left[s_{2}, t_{2}\right]$. If there exist some $\varepsilon_{i} \in\left(s_{i}, t_{i}\right), i=1,2, G(t, s)$ satisfying (i)-(ii) and a positive function $\rho \in C^{1}\left(\left[t_{0}, \infty\right), \mathbb{R}^{+}\right)$such that the inequalities (2.30) and (2.31) holds for $i=1,2$. Then Eq. (2.32) is oscillatory.

Remark 1 . If the hypotheses on the function $e(t)$ in condition (2.3) is replaced by the following condition

$$
e(t) \geq 0 \text { for } t \in\left[s_{1}, t_{1}\right] \text { and } e(t) \leq 0 \text { for } t \in\left[s_{2}, t_{2}\right]
$$

we will find the condition of the all above theorems and corollaries are valid as well.

Example 1. Consider the equation

$$
\left(t^{3 \lambda+1} x^{\prime}(t)\right)^{\prime}+p(t) x(t)\left(x^{\prime}(t)\right)^{2}+M t^{3 \lambda} x(t)=\sin t
$$

where $t \geq t_{0}>1, M>0, \lambda>0$ and $p(t)>0$ is any function. It is easy to verify that the conditions $\left(B_{1}\right)-\left(B_{3}\right)$ hold for the functions

$$
k_{1}(u, v)=v, k_{2}(u, v)=u v, f(u)=u
$$

for $\alpha=\beta_{1}=1$.

Moreover let $H(t)=t^{-\lambda} \sin t$ and $s_{1}=k \pi, t_{1}=(k+1) \pi, s_{2}=(k+1) \pi, t_{2}=$ $(k+2) \pi$. Then we have

$$
A_{s_{i}}^{t_{i}}(q ; 3)=\int_{s_{i}}^{t_{i}}|H(t)|^{3} q(t) d t=M \int_{s_{i}}^{t_{i}} \sin ^{6} t d t=\frac{5 M}{16} \pi, \text { for } i=1.2 .
$$

On the other hand,

$$
\begin{aligned}
A_{s_{1}}^{t_{1}}\left(\delta r\left|H^{\prime}\right|^{\alpha+1} ; 1\right) & =A_{s_{2}}^{t_{2}}\left(\delta r\left|H^{\prime}\right|^{\alpha+1} ; 1\right) \leq \frac{9}{4} \int_{\pi}^{2 \pi}\left(\lambda^{2} t^{-1}+4 t-4 \lambda \sin ^{5} t \cos t\right) d t \\
& =\frac{27}{2} \pi^{2}+\frac{9}{4} \lambda^{2} \ln 2
\end{aligned}
$$

So, the inequality (2.4) hold for $M>\frac{9}{10 \pi}\left(6 \pi^{2}+\lambda^{2 c} \ln 2\right)$. Thus Eq. (2.33) is oscillatory if $M>\frac{9}{10 \pi}\left(6 \pi^{2}+\lambda^{2} \ln 2\right)$ by Theorem 1 .

Example 2. Consider the equation

$$
\left(t^{3 \lambda+1} \frac{x^{\prime}(t)}{1+\left[x^{\prime}(t)\right]^{2}}\right)^{\prime}+\frac{p(t) x(t)\left(x^{\prime}(t)\right)^{2}}{1+\left[x^{\prime}(t)\right]^{2}}+N t^{3 \lambda} x(t)(\cos x(t)+2)=\sin t,
$$


where $t \geq t_{0}>1, N>0, \lambda>0$ and $p(t)>0$ is any function. Where

$$
k_{1}(u, v)=\frac{v}{1+v^{2}}, k_{2}(u, v)=\frac{u v}{1+v^{2}} \text { and } f(u)=u(\cos u+2) .
$$

It is easy to verify that the conditions $\left(B_{1}\right),\left(B_{3}\right),\left(B_{5}\right)$ and $\left(B_{6}\right)$ hold for for $\alpha=$ $\beta_{3}=K=\gamma=1$. It is easy to check that $Q=q$ for $K=\gamma=1$. Thus, by a similar calculating we can show that if $M>\frac{9}{10 \pi}\left(6 \pi^{2}+\lambda^{2} \ln 2\right)$ then Eq. (2.34) is oscillatory by Theorem 2 .

\section{REFERENCES}

[1] B. Ayanlar and A. Tiryaki, "Oscillation theorems for nonlinear second order differential equation with damping," Acta Math. Hungar., vol. 89, pp. 1-13, 2000, doi: 10.1023/A:1026716923088.

[2] D. Çakmak and A. Tiryaki, "Comment on the paper "oscillation of second-ordernonlinear ode with damping"," Applied Mathematics and Computation, vol. 191, p. 298, 2007, doi: 10.1016/j.amc.2007.02.059.

[3] S. R. Grace and B. S. Lalli, "Oscillation theorems for second order super linear differential equations with damping," J. Austral. Math. Soc. Ser. A, vol. 53, pp. 156-165, 1992.

[4] G. H. Hardy, J. E. Littlewood, and G. Polya, Inequalities, 2nd ed. Cambridge: Cambridge University Press, 1998.

[5] M. Kirane and Y. V. Rogovchenko, "Oscillation results for a second order damped differential equation with nonmonotonous nonlinearity," J. Math. Anal. Appl., vol. 250, pp. 118-138, 2000, doi: 10.1006/jmaa.2000.6975.

[6] M. Kirane and Y. V. Rogovchenko, "On oscillation of nonlinear second order differential equation with damping term," Applied Mathematics and Computation, vol. 117, pp. 177-192, 2001, doi: 10.1016/S0096-3003(99)00172-1.

[7] H. J. Li, "Oscillation criteria for second order linear differential equations," J. Math. Anal. Appl., vol. 194, pp. 217-234, 1995, doi: 10.1006/jmaa.1995.1295.

[8] W. Li and R. Agarwal, "Interval oscillation criterion for second order nonlinear differential equations with damping," Comp. Math. Appl., vol. 40, pp. 217-230, 2000, doi: 10.1016/S08981221(00)00155-3.

[9] F. Meng and Y. Huang, "Oscillation of second-order nonlinear ode with damping," Applied Mathematics and Computation, vol. 199, pp. 644-652, 2008, doi: 10.1016/j.amc.2007.10.025.

[10] F. Meng and Y. Huang, "Oscillation criteria for forced second-order nonlinear differential equations with damping," Journal of Computational and Applied Mathematics, vol. 224, pp. 339-345, 2009, doi: 10.1016/j.cam.2008.05.002.

[11] F. Meng and Y. Huang, "Interval oscillation criteria for a forced second-order nonlinear differential equations with damping," Applied Mathematics and Computation, vol. 218, pp. 1857-1861, 2011, doi: 10.1016/j.amc.2011.06.069.

[12] O. G. Mustafa and S. P. Rogovchenko, "Oscillation of nonlinear second order differential equations with damping term," J. Math. Anal. Appl., vol. 298, pp. 604-620, 2004, doi: 10.1016/j.jmaa.2004.05.029.

[13] S. P. Rogovchenko and Y. V. Rogovchenko, "Oscillation of second order differential equations with damping," Dynam. Contin. Discrete Impuls. Syst. Ser. A, vol. 10, pp. 447-461, 2003.

[14] S. P. Rogovchenko and Y. V. Rogovchenko, "Oscillation theorems for differential equations with a nonlinear damping term," J. Math. Anal. Appl., vol. 279, pp. 121-134, 2003, doi: 10.1016/S0022247X(02)00623-6.

[15] Y. V. Rogovchenko, "Oscillation theorems for second order equations with damping," Nonlinear Anal., vol. 41, pp. 1005-1028, 2000, doi: 10.1016/S0362-546X(98)00324-1. 
[16] N. Shang and H. Qin, "Comments on the paper: "oscillation of second-order nonlinear ode with damping" [applied mathematics and computation 199 (2008) 644-652]," Applied Mathematics and Computation, vol. 218, pp. 2979-2980, 2011, doi: 10.1016/j.amc.2011.07.064.

[17] A. Tiryaki and A. Zafer, "Oscillation criteria for second order nonlinear differential equation with damping," Turkish J. Math., vol. 24, pp. 185-196, 2000.

[18] A. Tiryaki and A. Zafer, "Oscillation of second order nonliner differential equations with nonlinear damping," Math. Comput. Modelling, vol. 39, pp. 197-208, 2004, doi: 10.1016/S08957177(04)90007-6.

[19] A. Tiryaki and A. Zafer, "Interval oscillation of a general class of second-order nonlinear differential equations with nonlinear damping," Nonlinear Anal, vol. 60, pp. 49-63, 2005, doi: 10.1016/j.na.2004.08.020.

[20] J. S. W. Wong, "On kamenev-type oscillation for second order differential equations with damping," J. Math. Anal. Appl., vol. 248, pp. 244-257, 2001, doi: 10.1006/jmaa.2000.7376.

[21] X. Yang, "Oscillation criteria for nonlinear differential equations with damping," Applied Mathematics and Computation, vol. 136, pp. 549-557, 2003, doi: 10.1016/S0096-3003(02)00079-6.

[22] X. Zhao and F. Meng, "Oscillation of second-order nonlinear ode with damping," Applied Mathematics and Computation, vol. 182, pp. 1861-1871, 2006, doi: 10.1016/j.amc.2006.06.022.

[23] Z. Zheng and F. Meng, "Oscillation criteria for forced second-order quasi-linear differential equations," Mathematical and Computer Modeling, vol. 45, pp. 215-220, 2007, doi: 10.1016/j.mcm.2006.05.005.

Authors' addresses

\section{Süleyman Öğrekçi}

Amasya University, Science \& Arts Faculty, Department of Mathematics, Ipekkoy, Amasya, Turkey

E-mail address: suleyman.ogrekci@amasya.edu.tr

Adil Mısır

Gazi University, Faculty of Science, Department of Mathematics, Teknikokullar, Ankara, Turkey

E-mail address: adilmegazi.edu.tr

Aydın Tiryaki

Izmir University, Faculty of Arts and Sciences, Department of Mathematics, Uckuyular, Izmir, Turkey

E-mail address: aydin.tiryaki@izmir.edu.tr 